\title{
О ДИНАМИКЕ КАНОНИЗИРУЕМЫХ СИСТЕМ ЛАГРАНЖА
}

\author{
(Представил Н. Алумяэ)
}

В работе, продолжающей исследования $\left[{ }^{1,2}\right]$, рассмотрена задача определения регуляторов и свойств управляемой системы, сводимой к канонической. Способом функциональной параметризации получены приводящие регуляторы и интегральные инварианты $I_{1}, I_{2 n}$ автономной системы. Показано, что ее решения - экстремали действия типа Якоби и Гамильтона при изопериметрическом условии. Предложен критерий канонизируемости исследуемых автономных и неавтономных систем.

1. Рассмотрим автономную систему Лагранжа в переменных Гамильтона с непотенциальными $\left(F \neq-\nabla_{q} V(q)\right)$ регуляторами

$$
\begin{array}{r}
x=Z\left(\nabla_{x} H+f^{-}\right)\left(x=\left(x_{i}\right)^{*}, x_{j}=q_{j}, x_{n+k}=p_{k}, x=d x / d t, \quad j, k=\overline{1, n}\right),(1.1) \\
Z=\left[\begin{array}{cr}
0 & 1_{n} \\
-1_{n} & 0
\end{array}\right], \quad \operatorname{det} Z=1, Z^{*}=Z^{-1}=-Z, \quad \triangle_{p} H(x) \stackrel{\Delta}{=}\left|\partial^{2} H / \partial p_{j} \partial p_{k}\right| \neq 0, \\
f^{-}=Z^{-1} f=-\left[\begin{array}{c}
F \\
0
\end{array}\right], \quad f=\left[\begin{array}{l}
0 \\
F
\end{array}\right], \quad F=\left(F_{j}\right)^{*}=F(x) \\
(i=\overline{1,2 n}, \quad j, k, s=\overline{1, n}) .
\end{array}
$$

Вид (1.1) имеют, в частности, автономные оптимальные системы $\left[{ }^{3}\right]$ $q=\partial H / \partial p, \quad p^{*}=-\partial H / \partial q+F, H=H(x)$ при $q=\left(q_{k}\right)^{*}, \quad p=\left(p_{s}\right)^{*}, \quad F=$ $=\Phi\left(x, u^{0}(x)\right)$ и неголономные системы Чаплыгина [ $\left.{ }^{1}\right]$, где $F_{s}=F_{s}-$ квадратичные формы $q_{s}^{*}$, а $F^{-}-$вектор гироскопических сил (1.1), причем

$-F_{j}^{-}=(\overbrace{\partial T_{2} / \partial q_{\alpha}^{*}})\left(\partial b_{s \alpha} / \partial q_{j}-\partial b_{j \alpha} / \partial q_{s}\right) q_{s}, \quad H=T_{2}-U(q)(\alpha=\overline{n+1, n+m})$,

$q=\left(q_{s}\right)^{*}, q_{\alpha}^{*}=g_{\alpha} \triangleq b_{s \alpha} q_{s}, \widetilde{T}_{2} \triangleq T_{2} \dot{q}_{\alpha}=g_{\alpha}$, а $b_{\text {s } \alpha}, T_{2}, U$ не зависяг от $t, q_{\alpha}$.

Известно $\left[{ }^{3}\right]$, что рассматриваемая оптимальная система, вообще говоря, не является канонической. Но в случае квадратичных по $q_{{ }_{k}}, q_{\alpha}{ }_{\alpha}$ форм $\widetilde{T}_{2}, T_{2}$ при $n_{1}=m=2$ в [ $\left.{ }^{1}\right]$ найдено условие на $T_{2}, b_{s \alpha}$, когда введение на траекториях (1.1), (1.2) нового функционального (неголономного) времени $\tau^{*}=\lambda, \tau[0]=0$ преобразует систему (1.1), (1.2) в каноническую

$\xi^{\prime}=Z \nabla_{\xi} G, \quad \xi=\left(q_{j}, P_{k}\right)^{*}, \quad \triangle_{P} G(q, P) \neq 0\left(v^{\prime}=\frac{d v}{d t}=\lambda^{-1} v^{*}\right)$. 


\section{В $\left[{ }^{1}\right]$ преобразование имеет вид}

$Q=q, \quad P=\lambda p, \quad G(q, P)=H\left(q, \lambda^{-1} P\right)=\tilde{H}, \quad \lambda=\lambda(q) \neq 0, \quad \lambda \in C_{1}\left(E^{n}\right)$, $\tau=\tau[t]=\int_{0}^{t} \lambda\left(q\left(\sigma, x_{0}\right)\right) d \sigma=\vartheta\left(t, x_{0}\right), \quad \forall \mid x_{0}=x(-t, x) \triangleq \Theta(t, x)\left(x_{0} \equiv x\left(0, x_{0}\right)\right)$.

Условие сводимости (1.1), (1.2) к (1.3) приводящей функцией $\lambda(q)$ эквивалентно требованию, чтобы система $(1.1),(1.2)$ имела [ $\left.{ }^{1}\right]$ множитель Якоби $M=\lambda(q)$. Простым примером скрытой каноничности является сводимая к (1.3) при $P:=\lambda^{*} p, G=\lambda^{*} H$ система

$$
\begin{gathered}
q=\nabla_{p} H, \quad p=-\nabla_{q} H+\Gamma \cdot q^{*}-H \nabla_{q} \mu^{*}, \quad \mu^{*}=\ln \left|\lambda^{*}\right|, \quad \lambda^{*}=\lambda^{*}(q) \neq 0, \\
\Gamma_{*}=\left[\gamma_{j k}^{*}\right], \quad \gamma_{j k}^{*}=p_{k} \partial \mu^{*} / \partial q_{j}-p_{j} \partial \mu^{*} / \partial q_{k}\left(W\left(F_{*}\right) \triangleq q^{*} \cdot F_{*} \neq\left(\lambda^{*} H\right) i=0\right) .
\end{gathered}
$$

В этой связи рассмотрим вопрос - когда система (1.1) (возможно, неавтономная) канонизируется локально обратимым преобразованием $T$ расширенного фазового пространства $x=x(\tau, \xi), t=t(\tau, \xi), t^{\prime} \neq 0$ ? Иными словами, установим на $H, F$ условия, при которых (1.1) как скрытая каноническая система обращается на $T$ в (1.3) и при которых не существует преобразования $T$, переводящего (1.1) в (1.3). В случае неприводимости (1.1) к (1.3) последняя является инвариантно неканонической относительно множества всех $T:=\{T\}$.

Для простоты ограничимся конфигурационным и двумя фазовыми подслучаями в подклассе преобразований $T_{0} \in\{T\}$ вида

$$
\tilde{\lambda} \in \Lambda: \tilde{\lambda}>0, \tilde{\lambda} \neq-q^{\prime} \cdot \nabla_{q} \tilde{\lambda}\left(\sim \lambda \neq P \cdot \nabla_{P} \lambda, \lambda(q, P)=\tilde{\lambda}\left(q, q^{\prime}\right)\right) \text {, }
$$

$q=Q, \quad p=p(q, P) \triangleq \psi(q, P): \nabla_{p} H=\lambda \nabla_{P} G, \quad \triangle_{P} G \neq 0\left(q=\lambda q^{\prime}, q^{\prime}=\nabla_{P} G\right)$,

$$
\tau^{\cdot}=\lambda, \quad \tau[0]=0 \rightarrow \tau=\Theta(t, x)=\tilde{\Theta}(t, \xi),\left.\left.\quad \Theta\right|_{t=0} \equiv \tilde{\Theta}\right|_{t=0} \equiv 0\left(\xi=\left[\begin{array}{c}
q \\
P
\end{array}\right]\right),
$$

заданном функциями $\lambda(q, P)$ (или $\tilde{\lambda} \in \Lambda)$ и $G(q, P)$ согласно условиям (1.5). Для этого подкласса канонический вид $q$-подсистемы сохраняется, преобразование $p, H$ в $P, G$ неканонично, автономно и включает преобразование $\left[{ }^{1}\right]$. В отличие от подхода $\left[{ }^{1}\right]$, мы определяем не $\lambda$ по $F$, а совокупность всех $F$, порожденных неголономным $\lambda$-преобразованием параметра-времени. Фиксируя допустимую порождающую функцию $G$, мы найдем соответственно выбору $\lambda=\lambda(q, P)$ эффект $t \rightarrow \tau$-параметризации в явном представлении регулятора $F$, что является мало изученным аспектом. При этом обобщим результаты [ $\left.{ }^{1}\right]$ на лагранжевы системы общего вида с $n>2$ и найдем их некоторые динамические характеристики и свойства. Введем обозначения

$$
\begin{aligned}
& L=q \cdot p-H, \quad \tilde{L}\left(q, q^{\prime}\right) \triangleq L\left(q, \lambda q^{\prime}\right), q^{\prime} \cdot P-G \triangleq K\left(q, q^{\prime}\right) \leftrightarrow G(q, P), \quad \text { (1.6) } \\
& R\left(q, q^{\prime}\right)=K-\widetilde{L}=\Delta L, r(q, P) \triangleq \nabla_{q} \cdot R, \quad q^{\prime} \cdot r-R \triangleq N(q, P)=G-H=\Delta H \text {, } \\
& p=\left.\nabla_{q} \cdot\right|_{q=g} \triangleq \psi(q, P), \quad q=\lambda \nabla_{P} G \stackrel{\triangleq}{=} g(q, P), \quad P=\left.\nabla_{q^{\prime}} K\right|_{q^{\prime}=h} \triangleq \varphi(q, p), \\
& \lambda p=P-r(q, P), q^{\prime}=\lambda_{*}^{-1} \nabla_{p} H \triangleq h(q, p), \lambda_{*}=\lambda_{*}(q, p)=\lambda(q, P)=\tilde{\lambda}\left(q, q^{\prime}\right) \text {, } \\
& q \leftrightarrow q^{\prime} \leftrightarrow P \leftrightarrow p, \quad q^{\prime}=\nabla_{P} G, \quad \mu=\ln \lambda ; \quad \lambda, \tilde{\lambda} \in \Lambda\left(q=\lambda q^{\prime}\right) .
\end{aligned}
$$


2. Рассмотрим конфигурационный случай $\lambda=\lambda(q)$. С учетом (1.6) из сводимости (1.1) к (1.3) с заданным гамильтонианом $G(q, P)$ и приводящей функцией $\lambda(q)$ преобразования (1.5) находим для $T_{0}$ структуру всех $F$, при которых (1.1) - скрытая каноническая система $F=F_{0}(q, p)=(q \cdot p) \nabla_{q} \mu-\left(q \cdot \nabla_{q} \mu\right) p-P_{s}^{\prime} \nabla_{P_{s}} r-\nabla_{q} N(q=\partial H / \partial p)$,

$$
P=\varphi(q, p)\left(a \cdot b=\sum_{s=1}^{n} a_{s} b_{s}=a_{s} b_{s}, \nabla_{v}=\partial / \partial v, \mu=\ln \lambda, q=d q / d t\right) .
$$

Здесь необходимо, чтобы (1.1) имела инвариант $G(q, \varphi(q, p))$ при каждой $F=F_{0}(q, p)$, заданной (2.1). Из уравнения мощности $W(F) \triangleq q^{\cdot} \cdot F$ следует, что регулятор $F_{0}$ не обязательно гироскопический

$$
W\left(F_{0}\right)=q \cdot F_{0}=-\lambda N^{\prime}=\left.H^{\cdot}\right|_{F=F_{0}} \not \equiv 0 .
$$

Подслучаем конфигурационного является вариант подобных по $p, P$ функций $H, G(G=\tilde{H} \triangleq H(q, \psi(q, P))$, где на общие лагранжевы системы с $n \geqslant 2$ обобщаются все результаты, полученные в [ $\left.{ }^{1}\right]$ способом приводящего множителя.

Здесь в силу (1.6) импульсы и лагранжианы подобны

$$
P=\lambda p, \quad K=\widetilde{L} \triangleq L\left(q, \lambda q^{\prime}\right)(N=G-H=0, R=0, r=0) .
$$

Из (2.1) все порожденные $\lambda(q)$ регуляторы приводимой системы

$$
F=\widetilde{F}_{0}=\Gamma q, \quad \Gamma=\nabla_{q} \mu p^{T}-p\left(\nabla_{q} \mu\right)^{T}, \quad W\left(F_{0}\right) \equiv 0
$$

являются гироскопическими, билинейными по $q ; p$ и имеют с $H$ одну степень по $p$. Согласно $(2.2)$, функция $H-$ инвариант (1.1) при $F=\Gamma q$. Приведем дополнительную информацию о динамике (1.1) с $\tilde{F}=\tilde{F}_{0}$. Из уравнения для множителя Якоби $\left[{ }^{4}\right]$ системы (1.1) в виде $M \cdot+M\left(\bigwedge_{p} F\right)=0$, где $\bigwedge_{v}=\underset{v}{\operatorname{div}}$, находим

$$
\bigwedge_{p} F_{0}=(1-n) \lambda^{-1} \lambda ; \quad d M / d \lambda=(n-1) \lambda^{-1} M \triangleq f(\lambda, M) .
$$

Следовательно, при $F=F_{0}$ (1.1) имеет зависящий от ее размерности множитель $M(q)=\lambda^{n-1}(q)$ и интегральный инвариант порядка $2 n$ вида $\left[{ }^{4}\right]$

$$
I_{2 n}=\int_{\Omega} \lambda^{n-1}(q) \delta q_{1} \ldots \delta q_{n} \delta p_{1} \ldots \delta p_{n}
$$

где $\Omega$ - произвольная $2 n$-мерная замкнутая область конечного объема. Для системы (1.1), (1.2) справедлива следующая

Л ем ма. Функция $\lambda=\lambda(q)$ будет приводящей для конфигурационного подкласса преобразований (1.5) с подобными $G, H$ лищь тогда, когда $\lambda^{n-1}(q)$ - множитель М системы (1.1), (1.2).

Для этого достаточно матричное равенство с $n(n-1) / 2$ независимыми условиями

$$
\begin{gathered}
\sum_{\alpha=n+1}^{n+m}\left(J_{\alpha}^{T}-J_{\alpha}\right) l_{\alpha}(p, q)= \\
=\nabla_{q} \mu^{T} p-p\left(\nabla_{q} \mu\right)^{T}\left(J_{\alpha}=\left[\frac{\partial b_{\alpha j}}{\partial q_{k}}\right], \quad l_{\alpha}=\left(\frac{\widetilde{T}}{\partial q_{\alpha}{ }_{\alpha}}\right)\right) .
\end{gathered}
$$


3амечание 1. Система (1.1) с $F=F_{0}(q, p)$ не имеет множителя Якоби $M_{1}$, являющегося ее инвариантом, в нетривиальном случае $\nabla_{q} \lambda \neq 0$. Действительно, из $\triangle_{p} H \nabla_{q} \lambda \neq 0$ следует $\nabla_{p} H \cdot \nabla_{q} \lambda=$ $=\lambda \neq 0$. Допуская противное, получаем, что $\lambda^{n-1}=I M_{1}-$ инвариант (1.1), (2.2), а это противоречит $\lambda \neq 0$ при $n>1$.

Система (1.1) при $F=F_{0}$ имеет интегральный неавтономный инвариант первого порядка

$$
\begin{gathered}
I_{1}=\oint_{c} \tilde{\omega}_{\delta}^{0}, \quad \tilde{\omega}{ }_{\delta}^{0}=\left(\lambda p-H \nabla_{q} \Theta\right) \cdot \delta q-H\left(\nabla_{p} \Theta \cdot \delta p+\frac{\partial \Theta}{\partial t} \delta t\right), \\
\tilde{\omega}_{\delta}^{0}=\lambda p \cdot \delta q-H \delta \Theta=P \cdot \delta q-G \delta \tau, \quad \xi=\left(q_{j}, P_{k}\right)^{*} \\
\Theta(t, x)=\tilde{\Theta}(t, \xi)=\int_{0}^{t} \lambda d t\left(\Theta \cdot=\frac{\partial \Theta}{\partial t}+(\Theta, H)+F \cdot \nabla_{p} \Theta=\lambda(x)\right),
\end{gathered}
$$

$\partial \Theta / \partial t+(\Theta, H)+F_{0} \cdot \nabla_{p} \Theta=\lambda(q),\left.\Theta\right|_{t=0} \equiv 0\left(\partial \Theta / \partial t=\lambda\left(x_{0}\right), x_{0}=x(-t, x)\right)$.

Функция $\tilde{\Theta}$ определяется при условии $\tilde{\Theta}(0, \xi) \equiv 0$ линейным уравнением

$$
\lambda^{-1} \partial \tilde{\Theta} / \partial t+(\tilde{\Theta}, G)=1 \quad\left(\partial \tilde{\Theta} / \partial t=\lambda[q(-t, \xi)]>0, \xi=\left(q_{k}, P_{\mathrm{s}}\right)^{*}\right),
$$

где $(\tilde{\Theta}, G)$ - скобка. Пуассона в переменных $\xi$, отвечающих $G$.

3 а м еч а и е 2. Коэффициенты дифференциальной формы $\tilde{\omega}_{d}^{0}$ можно получить из ее выражения в переменных है с учетом равенств (1.5), представленных расширенным каноническим преобразованием $T_{C}$ :

$P \cdot d Q-G d \tau=v(p \cdot d q-H d t)-d V$, если $V=V^{0}(t, Q, p) \in\{V\}$ при $v=1$. Из равенства нулювнешней производной $\tilde{\omega}_{d}{ }^{0}$ на союзных ей уравнениях (1.1), (2.2) при $\tau=\lambda$ следует (2.3).

3 а мечание 3 . Нужно различать системы (1.1) при $F=F_{0}$ и (1.4), совпадающие на их инвариантном множестве $H=0$, где движения системы $(1.1),(2.2)$ можно интерпретировать как решения канонической системы $\xi=Z \nabla_{\xi}\left(\lambda^{*} H\right)$ в многообразии $H=0$ с учетом $P=\lambda^{*} p$. Интегральный инвариант $I_{1}{ }^{*}$ системы (1.4) получаем из (2.3) заменой $\delta \tau$ на $\lambda^{*} \delta t$ в виде $I_{1}^{*}=\oint_{c} \omega_{\delta}^{*}, \omega_{\delta}^{*}=\lambda^{*}(p \cdot \delta q-H \delta t)$. Используя (1.5), (2.4) и расширенное каноническое преобразование $T_{C}$, сохраняющее автономность (1.3), получим в $T_{0}$ подмножество $T_{0}{ }^{*}$, приводящее $(1.4) \mathrm{K}$ канонической системе (1.3). В частности, если $\triangle_{P} G^{0} \neq 0$, то приводящая функция $\lambda=\lambda^{0}(q, P)$ и отображение $T_{0}{ }^{0}$ из $\left\{T_{0}{ }^{*}\right\}$ имеют вид $\lambda^{0}=\exp \mu^{0}, \quad \mu^{0}=\left(\lambda^{*} H\right)^{-1}, \quad G=G^{0}(q, P)=1 / 2\left(\lambda^{*} H\right)^{2}, \quad P=\lambda^{*} p, \quad d \tau^{0} / d t=\lambda^{0}$.

В связи с замечанием 3 рассмотрим фазовый случай $\lambda:=\tilde{\lambda}\left(q, q^{\prime}\right)$, учитывая (1.5), (1.6). Для простоты ограничимся двумя подслучаями. В первом из них предположим подобие $L, K$ по $q, q^{\prime}$ и $H, G$ по $p, P$, когда

$$
K=\widetilde{L}\left(q, q^{\prime}\right) \triangleq L\left(q, \lambda^{-1} q\right), \quad G(q, P) \triangleq H(q, \psi(q, P))
$$


С учетом (1.6), (2.5) для этого подслучая необходимо и достаточно, чтобы приводящая функция $\tilde{\lambda}_{0}$ преобразования (1.5) не зависела от $\left|q^{\prime}\right|$, т. е. была нуль-однородной по $q^{\prime}$ (или $q$ )

$$
\begin{gathered}
\tilde{\lambda}=\tilde{\lambda}_{0}(q, e)=\tilde{\lambda}_{0}\left(q, q^{*}\right)=\tilde{\lambda}_{0}\left(q, q^{\prime}\right)\left(q \cdot \nabla_{q} \tilde{\lambda}_{0} \equiv 0, e=\left|q^{\cdot}\right|^{-1} q^{*}\right), \\
\tilde{\mu}=\tilde{\mu}_{0}\left(q, q^{\prime}\right)=\tilde{\mu}_{0}(q, q)=\ln \tilde{\lambda}_{0}, \\
\nabla_{q^{\prime}} \tilde{\mu}_{0}=\tilde{\lambda}_{0} \nabla_{q} \cdot \tilde{\mu}_{0}\left(\partial^{2} K / \partial q^{\prime}{ }_{j} \partial q_{k}^{\prime}=\tilde{\lambda}_{0}^{2} \partial^{2} L / \partial q_{j} \partial q_{k}\right) .
\end{gathered}
$$

Величины $\sigma=q \cdot p=q^{\prime} \cdot P$ и $\tilde{\lambda}_{0}, \tilde{\mu}_{0}-$ дифференциальные инварианты (1.5), (2.5). Из (1.5), (1.6), (2.5), (2.6) -получаем связь $p \leftrightarrow P$ и заданную потенциалами $H, \tilde{\mu_{0}}$ вектор-функцию $F=F_{1}$ в этом подслучае

$$
\begin{gathered}
p=\psi(q, P)=\tilde{\lambda}_{0}^{-1}\left(P-\sigma \nabla_{q^{\prime}} \tilde{\mu}_{0}\right), \quad P=\varphi(q, p)=\tilde{\lambda}_{0}\left(p+\sigma \nabla_{q} \tilde{\mu}_{0}\right), \\
F_{1}(q, p)=\sigma\left(E\left[\mu_{0}\right]-\nabla_{q^{\prime}} \tilde{\mu}_{0}\right)-\tilde{\lambda}_{0}^{\prime} p, \quad E \stackrel{\Delta}{=} \nabla_{q}-\frac{d}{d t^{0}} \nabla_{q^{\prime}}, \\
\nabla_{q} \tilde{\mu}_{0}(q, q)=\nabla_{q} \tilde{\mu}_{0}\left(q, q^{\prime}\right), \quad \nabla_{q^{\prime}} \tilde{\mu}_{0} \triangleq \tilde{v}_{0}(q, P), \quad \tilde{\lambda}_{0}^{\prime}=\left(\tilde{\lambda}_{0}, G\right) .
\end{gathered}
$$

Легко убедиться, что приводимая система $(1.1)$, (2.7) имеет инвариант $H$, а регулятор $F_{1}-$ гироскопический: $W\left(F_{1}\right) \equiv 0$. Выражение $F_{1}(q, p)$ в $(2.7)$ находим из (1.3) заменой $\nabla_{q} \tilde{\mu}_{0}$ на $\tilde{v_{0}}$ и $P$ на $\varphi(q, p)$.

Рассмотрим в (1.5) подслучай подобия $P=\lambda p$, где $p \cdot q=$ $=P \cdot q^{\prime}=\sigma$ - дифференциальный инвариант преобразования. Обозначим $H\left(q, \lambda^{-1} P\right)=\mathscr{H}(q, P)$. Пусть $\tilde{H}$ и $\lambda \in \Lambda_{1}$ удовлетворяют условиям $\mu=V(\sigma), \triangle_{P}(\tilde{H}+\mu \sigma) \neq 0\left(\sigma=v(\mu), \sigma \equiv v(V(\sigma)), \mu=\ln \lambda, \Lambda_{1} \in \Lambda\right)$, (2.8)

$$
l[\tilde{H}]=v(\mu)(1-l[\mu]), d v / d \mu \neq 0 \quad\left(l[\Phi] \triangleq P . \nabla_{P} \Phi, 1-l[\mu] \neq 0\right),
$$

необходимым и достаточным для существования этого случая, причем $\triangle_{P} G \neq 0$. Из (2.8) и условий приводимости системы (1.1) к (1.3) находим

$$
\begin{gathered}
H(q, P)=H_{0}(q, e)+\int_{0}^{\mu} v(s) d s-\int_{0}^{\rho} v[\mu(q, \varrho, e)] d_{\varrho}, \\
G(q, P)=G_{0}(q)+\int_{0}^{\mu} v(s) d s+\not \quad\left(e=|P|^{-1} P, \varrho=\ln |P|\right),
\end{gathered}
$$

где $G_{0}(q), H_{0}(q, e), v(\mu)$ - произвольные достаточно гладкие функции $(d v / d \mu \neq 0)$. С учетом (1.3), (2.8), (2.9) заданный функциями $v, H$ регулятор $F_{2}$ имеет при $G \equiv 0$ структуру и мощность

$$
F_{2}=-\mu^{\prime} p, \quad W\left(F_{2}\right)=-v(\mu) \mu^{\prime} \neq \equiv \quad\left(\mu^{\prime}=\left.(\mu, G)\right|_{\xi \rightarrow x}\right) .
$$

В этом подслучае необходимо, чтобы $G(q, \lambda(\sigma) p) \equiv 0$ в силу (1.1), (2.10).

Во всех рассмотренных случаях система (1.1) с $F=F_{\alpha}(\alpha=\overline{0,2)}$ имеет инвариант $G(q, \varphi(q, p))$ и интегральные инварианты.

$$
I_{1}=\oint_{c}\left[\varphi^{\tau}(q, p) \circ \delta q-G \delta \Theta\right]\left(\left.\Theta(t, x) \stackrel{\Delta}{=} \vartheta\right|_{x_{0}=x(-t, x)}, \vartheta \stackrel{\Delta}{=} \int_{0}^{t} \lambda\left[x\left(t, x_{0}\right)\right] d t\right) \text {, }
$$




$$
\begin{gathered}
I_{2 n}=\int_{\Omega} M(x) \delta q_{1} \ldots \delta q_{n} \delta p_{1} \ldots \delta p_{n} \\
\left(M=\lambda_{*}^{-1} \operatorname{det}[\partial \varphi / \partial p], \varphi(q, p)=P, x=\left(q_{j} p_{k}\right)^{*}\right) .
\end{gathered}
$$

При этом $q$-компонента траектории (1.1) сохраняется для (1.3), a ее импульс преобразуется силами $F_{\alpha}$ функциональной параметризации по закону $p \rightarrow P=\varphi(q, p)$. Решения (1.1) при $F=F_{\alpha}$ находим интеграцией (1.3) и обращением интеграла

$t=\int_{0}^{\tau} \lambda^{-1}(q[\tau], P[\tau]) d \tau\left(\lambda(q, P) \triangleq \lambda_{*}(q, p)=\tilde{\lambda}_{*}(q, q), \quad P=\varphi\left(q, \nabla_{q} L\right) \stackrel{\Delta}{=} \tilde{\varphi}\right)$.

Из (1.1) $\left(F=F_{\alpha}\right)$ и (1.3) заключаем, что $q[t]$ доставляет стационарное значение действию

$$
S=\int_{0}^{t_{1}} \tilde{\lambda} \cdot\left[\left(P \cdot \nabla_{P} G\right)-P\right] d t, \text { где } P=\tilde{\varphi}\left(q, q^{*}\right),
$$

при условиях $\tau_{1}=\int_{0}^{t_{1}} \tilde{\lambda} . d t=$ const, $q[0]=q_{0}=$ const, $q\left[t_{1}\right]=q_{1}=$ const.

Траектории (1.1) $F=F_{\alpha}$ в координатном пространстве $q$ находим из стационарности функционала $S$. типа Якоби

$$
\begin{array}{r}
S_{*}=\int_{q_{n}}^{q_{n t}} \sigma_{*}\left(q, \tilde{q}^{\prime}, g_{0}\right) d q_{n}\left(\sigma_{*}=h_{*}^{-1}\left[K\left(q, h_{*} \tilde{q}^{\prime}, h_{*}\right)+g_{0}\right], \dot{\tilde{q}^{\prime}}=\left(\frac{d q_{s}}{d q_{n}}\right)^{*},\right. \\
s=\overline{1, n-1}),
\end{array}
$$

где использованы обозначения $(1.3),(1.5),(1.6)$ и $h$. - решение уравнения $G_{*}\left(q, h_{*} \tilde{q}^{\prime}, h_{*}\right)=g_{0}=$ const, $G_{*}\left(q, q^{\prime}\right) \stackrel{\Delta}{=} G(q, P)$. Уравнение времени имеет вид

$$
t-t_{0}=\int_{q_{n}}^{q_{n 1}}\left(\chi h_{*}\right)^{-1} d q_{n}\left(\chi \triangleq \tilde{\lambda}\left(q, h_{*} \tilde{q}^{\prime}, h_{*}\right), q_{n}^{\prime}=h_{*}, t_{0}=\tau_{0}, \tau[0]=0\right) .
$$

В силу (2.11) влияние $F=F_{\alpha}$ на геометрию движений (1.1) в $q$-пространстве сводится к замене $L, H$ на $K, G$ согласно (1.5), (1.6).

3. Условия канонизируемости автономных и неавтономных систем (1.1) определим следующим критерием.

Т еорем а. Для сводимости системы (1.1) $\kappa$ (1.3) расширенным фазовым преобразованием $T$ необходимо и достаточно, чтобы (1.1) имела интегральный инвариант $I_{1}$ с динамическим пфаффианом $\omega_{d}-$ неособенной формой Пфаффа класса $2 n+1 u \operatorname{det} R\left[\omega_{d}\right] \neq 0$.

Здесь

$$
I_{1}=\oint_{C} \omega_{\delta}, \quad \omega_{d} \triangleq g \cdot d x-h d t\left(g=g(t, x)=\left(g_{i}\right)^{*}, \quad h=h(t, x) \in C_{1}\right),
$$

$d I_{1} / d s=0, d t / d s=v(t, x)-$ произвольная функция класса $C_{1}, v \neq 0$,

$$
R\left[\omega_{d}\right]=R=\left[r_{i \sigma}\right], \quad r_{i \sigma}=\partial g_{i} / \partial x_{\sigma}-\partial g_{\sigma} / \partial x_{i}, k=\partial g_{i} / \partial t-\partial h / \partial x_{i}
$$

$$
\left(R^{*}=-R, \quad i, \sigma=\overline{1,2 n}\right) \text {. }
$$


Н еобходим ость. Если существует преобразование $T\left(t^{\prime} \neq 0\right)$ системы (1.1) в (1.3), то последняя имеет интегральный инвариант Пуанкаре-Картана

$$
T_{1}=\underset{\ddot{C}}{\oint \omega_{\delta},} \quad \tilde{\omega} d \stackrel{\Delta}{=} P \cdot d Q-G d \tau\left(d I_{1} / d s=0, d \tau / d s=\widetilde{v}(\tau, \xi), \quad \tilde{v}=t^{\prime} v\right) \text {, }
$$

где $\tilde{\omega}_{d}-$ динамическая форма Пфаффа. Из сохранения значения $I_{1}=I_{1}$ на преобразовании $T$ и $t^{\prime} \neq 0$ следует, что система (1.1) имеет интегральный инвариант (3.1) с динамическим пфаффианом $\omega_{d}=\tilde{\omega_{d}}$ в переменных $t, x$ и $C=T(\tilde{C})$.

Д ост а точность. Пусть система (1.1) имеет инвариант (3.1). Тогда в силу теоремы Пфаффа $\left[{ }^{5}\right]$ существует преобразование $\widetilde{T} \in\{T\}$ $(d t / d \tilde{\tau} \neq 0)$, приводящее $\omega_{d}$ в новых переменных $\tilde{\tau}, \tilde{\xi}$ к каноническому виду $\omega_{d}{ }^{0}=\widetilde{P} \cdot d \widetilde{Q}-d \tilde{\tau}$. Расширенным каноническим преобразованием $T_{C} \in\{T\}$ из $\omega_{d}^{0}$ получаем $\tilde{\omega} d$, где $\Delta_{P} G \neq 0$. Но союзные $\varepsilon$ формой (3.2) уравнения Пфаффа - каноническая система (1.3). Следовательно, преобразование $T=\widetilde{T} \cdot T_{C}$ переводит систему (1.1) в (1.3). Теорема доказана.

Сл ед с т и е 1 . Условие $d I_{1} / d s=0$ в (3.1) приводит к системе Пфаффа $R x^{*}=k$. Отсюда при заданных $H, F$ для сводимости (1.1) к (1.3) необходимо и достаточно, чтобы существовали $2 n+1$ функций $g(t, x)$, $h(t, x)$ динамического пфаффиана $\omega_{d}$, удовлетворяющих совместной системе [ $\left.{ }^{4}\right]$ из $2 n$ линейных однородных уравнений

$R Z\left(\nabla_{x} H+f^{-}\right)=k, \quad$ где $\quad k \neq 0\left(\operatorname{det} R Z \neq 0, \triangle_{p} H \neq 0 \rightarrow \nabla_{p} H \neq 0\right)$,

$B=\left[\begin{array}{l}B_{1} \\ B_{2}\end{array}\right] \triangleq(R Z)^{-1}=-Z R^{-1}, \quad B_{\beta}-$ матрицы порядка $n \times 2 n$ и ранга $n$ $(\beta=1,2)$.

Пусть функция $H(t, x)$ системы (1.1) задана, $\omega_{d}-$ динамический пфаффиан, а вектор-функция $\left(h, g_{i}\right)^{*}$ от $t, x$ удовлетворяет $n$ уравнениям $B_{2} k=\nabla_{p} H$. С учетом (3.1), (3.3) заключаем, что множество $F$, на котором (1.1) является скрытой канонической системой, имеет вид $F=\nabla_{q} H-B_{1} k$. Если эти равенства не выполнены, то система (1.1) будет инвариантно неканонической на $\{T\}$. Этот результат - решение общей задачи раздела 1.

Следстви е 2. Пусть $u^{0}(t, x)$ - регулятор (1.1), оптимальный по интегральному $\left[{ }^{3}\right]$ критерию $I[u] \rightarrow \min _{u}$ при условии $d I_{1} / d s=0$, где $F \triangleq \Phi(t, x, u), u \in U, \omega_{d}-$ заданный динамический пфаффиан интегрального инварианта (3.1). Тогда вообще неавтономная оптимальная система (1.1) из раздела 3 будет скрытой канонической.

\section{ЛИТЕ РАТ УРА}

1. Чаплыгин С. А., Исследования по динамике неголономных систем, М.-Л., ГИТТЛ, 1949 , с. $28-38$.

2. Биркгоф Дж. Д., Динамические системы, М.-Л., ОГИЗ, гиттЛ, 1941, c. $34-54$. 
3. Г ель фанд И. М., Фомин С. В., Вариационное исчисление, М., ГИФМЛ, 1961, c. $210-228$.

4. Г урс а Э., Курс математического анализа, т. 2, М.-Л., ОНТИ, 1936, с. 341-346, $510-532$.

5. Р а ше в ски й П. К., Геометрическая теория уравнений с частными производными, М.-Л., ОГИЗ, Гостехиздат, 1947, с. 142-168.

Институт кибернетики

Академии наук Эстонской ССР

Поступила в редакцию 29/XII 1980

\section{KEIS}

\section{KANONISEERITAVATE LAGRANGE'I SUSTEEMIDE DUNAAMIKAST}

Artiklis on analüüsitud Lagrange'i süsteemi omadusi ja kanoniseerimise meetodit. On esitatud autonoomsetele süsteemidele sobiv lahendusvôte, mis põhineb funktsionaalsel ajateisendusel ja genereeriva potentsiaali kasutamisel. Lõpptulemusena on saadud mitmesugused regulaatorid ning dünaamilised ja integraalsed invariandid. Olalmainitud süsteemide jaoks on tuletatud Hamiltoni ja Jacobi printsiipide üldistused ning tõestatud üldiste (nii autonoomsete kui ka mitteautonoomsete) süsteemide ühise kanoniseerimise kriteerium.

\section{KEIS}

\section{ON THE DYNAMICS OF THE LAGRANGE SYSTEMS REDUCIBLE TO THE CANONICAL FORM}

The problem of the canonical representation and the corresponding properties of the system noted in the headline are investigated in this paper. A new way based on the transform $\tau=\lambda(q, P)$ and the generating potential $G=G(q, P)$, is introduced and applied to the autonomous systems. As a result, necessary controls $F=F_{\alpha}(q, p)$ $(\alpha=\overline{0,2})$ are derived due to the appropriate classification covering various essentially new cases. Under the defined assumptions autonomous systems obtain dynamical and integral invariants

$G[q, \varphi(q, p)], \quad I_{1}=\oint_{c} \varphi(q, p) \cdot \delta q-G \delta \Theta, \quad I_{2 n}=\int_{\Omega} \lambda_{*}^{-1}\left|\frac{\partial \varphi}{\partial p}\right| \delta q_{1} \ldots \delta q_{n} \delta p_{1} \ldots \delta p_{n}$.

For these systems the generalized Hamilton and Jacobi principles are obtained. For the autonomous and non-autonomous systems criterion of reducibility to the canonical form is proposed in the theorem.

The principal statement of the problem is as follows. The transform $T$ leads (1.1) to the system (1.3), if the conditions (3.1) are valid.

Hence, if the controls $(2.1),(2.7),(2.10)$ are applied to the relevant subsystems (1.6), (2.5), (2.8), the transform (1.5) reduces the initial system (1.1) to the canonical form. 